\title{
Technical Note: Adaptation of an Animal-Model Method for Approximation of Reliabilities to a Sire-Maternal Grandsire Model
}

\author{
G. R. Wiggans, ${ }^{* 1}$ S. Tsuruta, $\dagger$ and I. Misztal† \\ *Animal Improvement Programs Laboratory, Agricultural Research Service, USDA, Beltsville, MD 20705-2350 \\ †Department of Animal and Dairy Science, University of Georgia, Athens 30602
}

\begin{abstract}
A method used to approximate reliabilities for animal models was modified to estimate reliabilities for sire-maternal grandsire (MGS) models. Accuracy of the approximation was tested on a calving-ease data set for 2,968 bulls for which the inverse of the coefficient matrix could be obtained. Correlations between estimated and true reliabilities ranged from 0.984 to 0.998 for first- and later-parity calving ease for sire and MGS effects. With no modification of the animal-model procedure, MGS identification was treated as if it were dam identification, which resulted in overestimated reliability. When pedigree information was ignored, reliability was underestimated. Correlations with true values were lower for both of those cases when compared with correct processing of MGS information. The modification provided a slight improvement over assuming MGS to be unknown and will be used for routine USDA evaluation of calving traits.
\end{abstract}

Key words: reliability, sire-maternal grandsire model

Sire-maternal grandsire (MGS) models are widely used for calving traits because of the smaller computational requirements compared with animal models (AM). Threshold models have been applied to calving traits but often are limited to 1 categorical trait. Low correlations between first- and later-parity performance for calving ease and stillbirth (Wiggans et al., 2006) have suggested that a multitrait model with separate traits for first and later parity should be used. With appropriate transformation of observations, a linear model can often approximate the results of a threshold model (Carlén et al., 2006). For routine evaluations that are used by the dairy industry, an indication of ac-

Received December 17, 2007.

Accepted June 16, 2008.

${ }^{1}$ Corresponding author: George.Wiggans@ars.usda.gov curacy is required. Therefore, a method of calculating reliability for a multitrait sire-MGS linear model was needed. Strabel et al. (2001) developed an AM method to approximate reliability that can accommodate multitrait models. Their procedure was modified for a sire-MGS model so that MGS identification (ID) could replace dam ID and be used correctly.

The inverse of the elementary numerator relationship matrix ( $\mathrm{A}^{-1}$; Henderson, 1975) among a bull and its sire and MGS as used in sire models is

$$
\mathbf{A}^{-1}=\left[\begin{array}{ccc}
16 / 11 & -8 / 11 & -4 / 11 \\
-8 / 11 & 4 / 11 & 2 / 11 \\
-4 / 11 & 2 / 11 & 1 / 11
\end{array}\right]
$$

Using the notation of Misztal and Wiggans (1988; equation [12]), contributions due to relationships among bull (B), sire (S), and MGS were expressed as

$$
\begin{gathered}
{\left[\begin{array}{ccc}
(16 / 11) \alpha+\mathrm{q}_{\mathrm{B}} & (-8 / 11) \alpha & (-4 / 11) \alpha \\
(-8 / 11) \alpha & (15 / 11) \alpha+\mathrm{q}_{\mathrm{S}} & (2 / 11) \alpha \\
(-4 / 11) \alpha & (2 / 11) \alpha & (12 / 11) \alpha+\mathrm{q}_{\mathrm{MGS}}
\end{array}\right]^{-1}} \\
=\left[\begin{array}{ccc}
1 /\left(\alpha+\mathrm{b}_{\mathrm{B}}\right) & * & * \\
* & 1 /\left(\alpha+\mathrm{b}_{\mathrm{S}}\right) & * \\
* & * & 1 /\left(\alpha+\mathrm{b}_{\mathrm{MGS}}\right)
\end{array}\right]
\end{gathered}
$$

where $\alpha$ is the ratio of error variance to bull genetic variance; $q$ is the number of effective daughters for the animal without relationships among the bull, sire, and MGS considered; $b$ is the number of effective daughters for the animal with relationships among the bull, sire, and MGS considered; and * indicates that the structure of the matrix element is unknown. The sire and MGS are considered to be unrelated and to have unknown parents. Their diagonal elements include an additional contribution of 1 to account for their contributions to themselves (Henderson, 1975). Then, based on Misztal and Wiggans (1988; equation [13]), solutions for $b$ are 


$$
\begin{aligned}
b_{\text {В }}= & (5 / 11) \alpha+q_{B}-\left\{[(8 / 11) \alpha]^{2}\left[(12 / 11) \alpha+q_{M G S}\right]\right. \\
& -2[(2 / 11) \alpha][(4 / 11) \alpha][(8 / 11) \alpha] \\
& \left.+[(4 / 11) \alpha]^{2}\left[(15 / 11) \alpha+q_{S}\right]\right\} / \\
& \left\{\left[(15 / 11) \alpha+q_{S}\right]\left[(12 / 11) \alpha+q_{M G S}\right]-[(2 / 11) \alpha]^{2}\right\} ;
\end{aligned}
$$$$
\mathrm{b}_{\mathrm{S}}=(4 / 11) \alpha+\mathrm{q}_{\mathrm{S}}-\left\{[(8 / 11) \alpha]^{2}\left[(12 / 11) \alpha+\mathrm{q}_{\mathrm{MGS}}\right]\right.
$$$$
-2[(2 / 11) \alpha][(4 / 11) \alpha][(8 / 11) \alpha]
$$$$
\left.+[(2 / 11) \alpha]^{2}\left[(16 / 11) \alpha+\mathrm{q}_{\mathrm{B}}\right]\right\} /
$$$$
\left\{\left[(16 / 11) \alpha+\mathrm{q}_{\mathrm{B}}\right]\left[(12 / 11) \alpha+\mathrm{q}_{\mathrm{MGS}}\right]-[(4 / 11) \alpha]^{2}\right\} ;
$$$$
\mathrm{b}_{\mathrm{MGS}}=(1 / 11) \alpha+\mathrm{q}_{\mathrm{MGS}}-\left\{[(4 / 11) \alpha]^{2}\left[(15 / 11) \alpha+\mathrm{q}_{\mathrm{S}}\right]\right.
$$$$
-2[(2 / 11) \alpha][(4 / 11) \alpha][(8 / 11) \alpha]
$$$$
\left.+[(2 / 11) \alpha]^{2}\left[(16 / 11) \alpha+\mathrm{q}_{\mathrm{B}}\right]\right\} /
$$$$
\left\{\left[(16 / 11) \alpha+q_{B}\right]\left[(15 / 11) \alpha+q_{S}\right]-[(8 / 11) \alpha]^{2}\right\} .
$$

The AM method for approximating reliability was tested for its performance with sire-MGS models. Solutions for sire and MGS effects were not calculated as part of the test because reliability is calculated separately. Full sire-MGS pedigrees were used for estimation of approximate reliabilities. A set of 36,238 calving-ease records with no missing observations was analyzed. Those records represented the 2,968 Holstein bulls with the most records (sire or MGS). The coefficient matrix in the sire-MGS model was inverted to provide true reliabilities. The model included ef- fects of first- and later-parity calving ease for sire and MGS. The AM method was tested in 4 configurations: 1) pedigree data were ignored, 2) MGS was assumed to be unknown (sire only), 3) MGS ID was incorrectly processed as though it was dam ID (no modification), and 4) MGS ID was accounted for correctly (modified AM method).

Incorrectly processing MGS ID as though it was dam ID caused reliability to be overestimated (Table 1). With no pedigree information, reliabilities were underestimated. Correlations between true and estimated reliability (Table 2) were lowest for both those configurations. Performance of the modified AM method was good across the range of reliabilities although bias increased with true reliability. Correlations ranged from 0.984 to 0.998 for the sire and MGS effects by parity (Table 2). The modification provided a slight improvement in the correlation between true and estimated reliability compared with assuming MGS to be unknown even though estimation error (Table 1) was slightly greater. The approximation method of Misztal and Wiggans (1988) does not account for the distribution of contemporary groups; therefore, accuracies are usually inflated. Ignoring relationships between sire and MGS also may contribute to accuracy overestimation. The loss in reliability when MGS is assumed to be unknown partly offsets the inflation.

Values for regression of estimated reliability on true reliability are in Table 3. Slopes with the modified AM method were between 1.02 and 1.10, which indicates a slight overestimation. The modified method had the smallest SD of estimation errors. The regression analysis confirmed that 1) assuming MGS to be unknown was slightly less accurate than including MGS correctly and 2) including no pedigree information or incorrectly treating MGS ID as dam ID was substantially less accurate.

Berger (1991) also found that approximated reliabilities for bull evaluations from a calving-ease threshold model with pedigree information included overesti-

Table 1. Mean true reliabilities and estimation error from 4 configurations for estimating reliability for 2,968 Holstein bulls

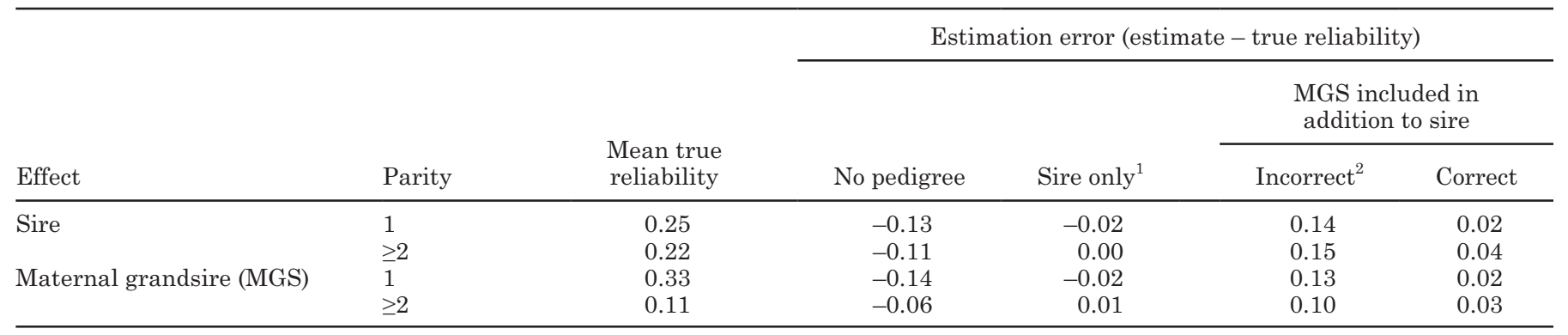

${ }^{1}$ Maternal grandsire considered to be unknown.

${ }^{2}$ Maternal grandsire included as dam. 
Table 2. Correlations between true reliability and estimates from 4 configurations for estimating reliability for 2,968 Holstein bulls

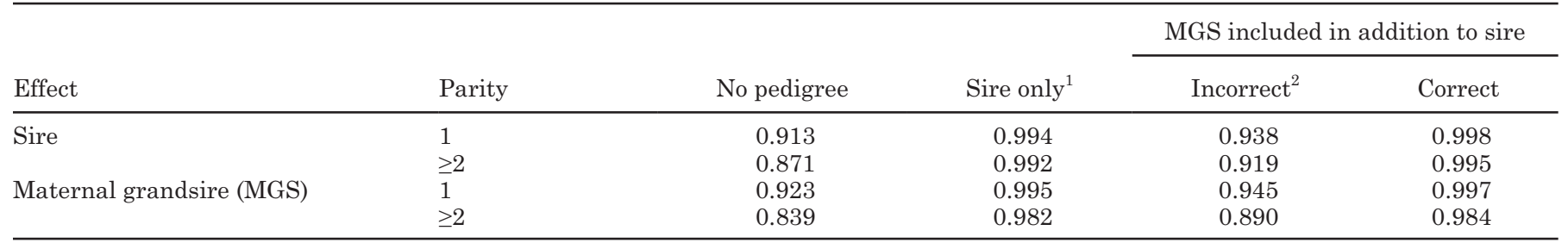

${ }^{1}$ Maternal grandsire considered to be unknown.

${ }^{2}$ Maternal grandsire included as dam.

mated true reliabilities slightly more (2.5 to $3.9 \%)$ than approximated reliabilities based on progeny data alone (0.0 to 1.4\%). However, the use of pedigree information increased accuracy of Berger's approximated reliabilities as indicated by reductions in mean square error of 16 and $69 \%$ for 2 data sets. Based on distributions of differences between predicted and expected reliabilities, Berger concluded that the greatest benefit from including pedigree information was for bulls that had a sire and MGS without progeny calving-ease records, but the number of bulls with a sire or MGS or both with progeny data was limited. In this study, the benefit of including pedigree information was nearly constant across reliabilities of sire and MGS evaluations, probably because most bulls had low reliabilities; therefore, true reliability contained a large contribution from sire and MGS reliabilities.

Estimation errors for the modified AM method were nearly normally distributed with a slightly larger tail for overestimation (Figure 1). Because of the low mean true reliabilities (Table 1), the opportunity for substantial underestimation was limited. Part of the overestimation was caused by ignoring how much information was provided by various relationships; for example, full sibs provide less information than half sibs. Sanchez et al. (2008) also found that approxima- tion algorithms overestimated evaluation accuracy. Both the modified multitrait and the random regression methods in that study overestimated accuracies of predicted breeding values for similar types of animals. Comparison of characteristics of bulls with estimation errors of $<0.01$ or $\geq 0.01$ showed little difference except that the group of bulls with low or negative bias was smaller and had lower true reliability than the group of bulls with greater positive bias.

Reliabilities for second parity and later were slightly more inflated than for first parity with the modified AM method (Tables 1 and 3 and Figure 1). Because of a greater number of first-parity records (with possibly greater heritability) than later-parity records, a larger portion of reliabilities for later parities was obtained indirectly through first-parity information. The algorithm of Strabel et al. (2001) includes information from correlated traits by an approximate method that combines single-trait reliabilities into multiple-trait reliabilities.

The method of Strabel et al. (2001) for approximating AM reliabilities can provide reasonable reliability estimates for a sire-MGS model either by assuming MGS to be unknown or by modifying the method to process MGS ID correctly in place of dam ID. The modified AM method was implemented by USDA (Beltsville, MD) to

Table 3. Regression of estimates from 4 configurations for estimating reliability on true reliability and SD of estimation errors for 2,968 Holstein bulls

\begin{tabular}{|c|c|c|c|c|c|c|}
\hline \multirow[b]{2}{*}{ Statistic } & \multirow[b]{2}{*}{ Effect } & \multirow[b]{2}{*}{ Parity } & \multirow[b]{2}{*}{ No pedigree } & \multirow[b]{2}{*}{ Sire only ${ }^{1}$} & \multicolumn{2}{|c|}{$\begin{array}{l}\text { MGS included in } \\
\text { addition to sire }\end{array}$} \\
\hline & & & & & Incorrect $^{2}$ & Correct \\
\hline \multirow{3}{*}{ Regression coefficient } & & $\geq 2$ & 0.999 & 1.114 & 1.022 & 1.096 \\
\hline & Maternal grandsire & 1 & 1.124 & 1.061 & 0.886 & 1.020 \\
\hline & & $\geq 2$ & 0.793 & 1.083 & 1.125 & 1.100 \\
\hline \multirow{2}{*}{ SD of estimation error } & Maternal grandsire & $\overline{1}$ & 0.102 & 0.023 & 0.067 & 0.016 \\
\hline & & $\geq 2$ & 0.037 & 0.015 & 0.042 & 0.014 \\
\hline
\end{tabular}

\footnotetext{
${ }^{1}$ Maternal grandsire (MGS) considered to be unknown.

${ }^{2}$ Maternal grandsire included as dam.
} 


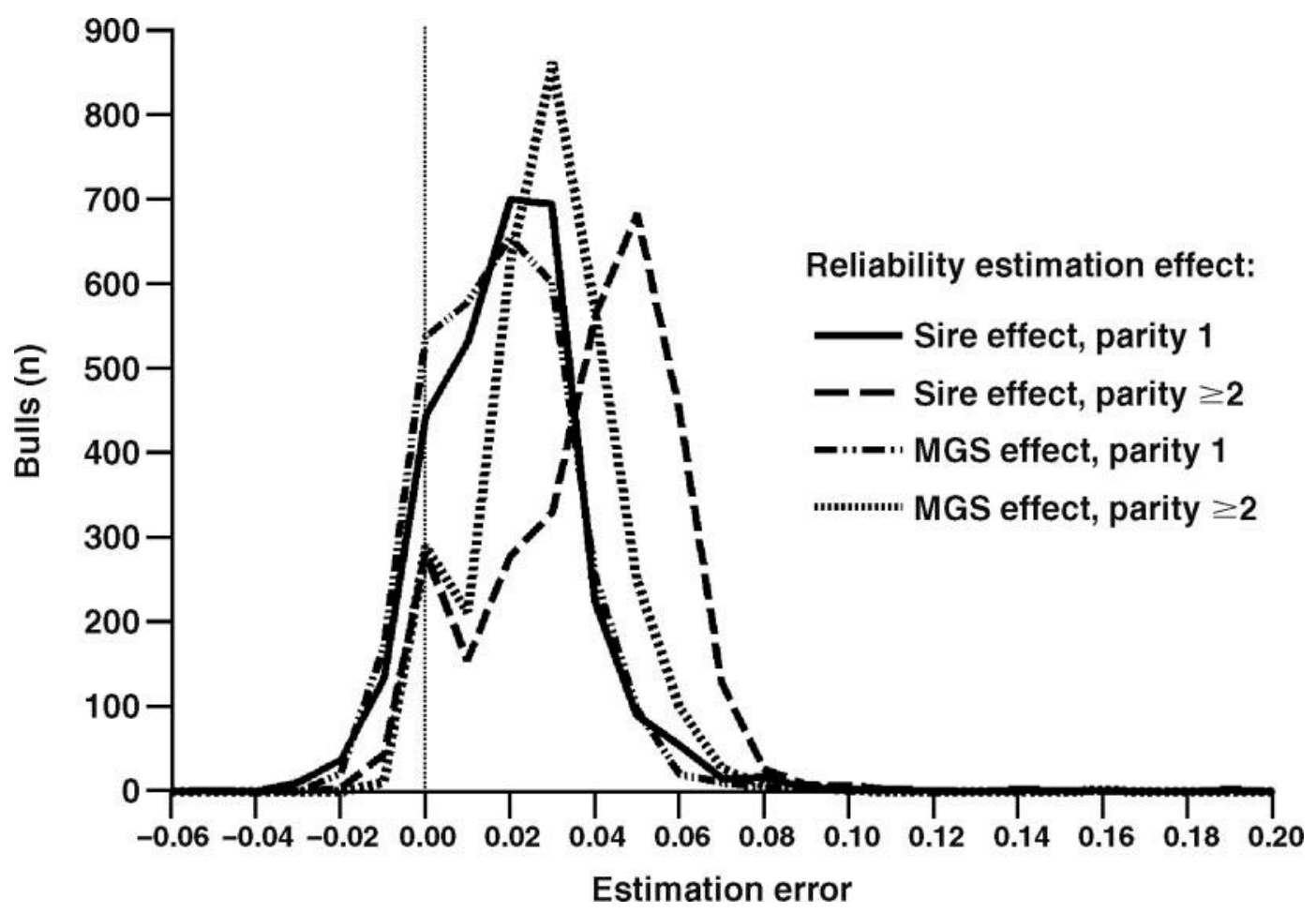

Figure 1. Distribution of estimation error (estimated - true reliability) for effects included in reliability estimation that correctly incorporates pedigree information from sire and maternal grandsire (MGS) for 2,968 Holstein bulls.

calculate reliabilities for routine evaluation of US calving traits in May 2008. The implementation used the Fortran 90 program accf90.f90, which can be obtained through a collaborative research agreement with I. Misztal (University of Georgia, Athens; Ignacy@uga. edu).

\section{REFERENCES}

Berger, P. J. 1991. Reliability of sire evaluations for calving ease by a threshold model analysis. J. Dairy Sci. 74:1069-1077.

Carlén, E., U. Emanuelson, and E. Strandberg. 2006. Genetic evaluation of mastitis in dairy cattle using linear models, threshold models, and survival analysis: A simulation study. J. Dairy Sci. 89:4049-4057.
Henderson, C. R. 1975. Inverse of a matrix of relationships due to sires and maternal grandsires. J. Dairy Sci. 58:1917-1921.

Misztal, I., and G. R. Wiggans. 1988. Approximation of prediction error variance in large-scale animal models. J. Dairy Sci. 71(Suppl. 2):27-32.

Sanchez, J. P., I. Misztal, and J. K. Bertrand. 2008. Evaluation of methods for computing approximate accuracies of predicted breeding values in maternal random regression models for growth traits in beef cattle. J. Anim. Sci. 86:1057-1066.

Strabel, T., I. Misztal, and J. K. Bertrand. 2001. Approximation of reliabilities for multiple-trait model with maternal effects. J. Anim. Sci. 79:833-839.

Wiggans, G. R., C. P. Van Tassell, J. B. Cole, and L. L. M. Thornton. 2006. Genetic correlations between first and later parity calving ease in a sire-maternal grandsire model. Proc. 8th World Congr. Genet. Appl. Livest. Prod., Commun. 01-92. 